\title{
Imaging VCAM-1 as an Indicator of Treatment Efficacy in Metastatic Ovarian Cancer
}

\author{
Jennifer M. Scalici ${ }^{1}$, Stephanie Thomas ${ }^{2}$, Christine Harrer ${ }^{3}$, Timothy A. Raines ${ }^{3}$, Joanna Curran ${ }^{3}$, Kristen A. Atkins ${ }^{4,5}$, \\ Mark R. Conaway ${ }^{5,6}$, Linda Duska ${ }^{1,5}$, Kimberly A. Kelly ${ }^{2,5}$, and Jill K. Slack-Davis ${ }^{3,5}$ \\ ${ }^{I}$ Department of Obstetrics and Gynecology, University of Virginia, Charlottesville, Virginia; ${ }^{2}$ Department of Biomedical Engineering, \\ University of Virginia, Charlottesville, Virginia; ${ }^{3}$ Department of Microbiology, Immunology and Cancer Biology, University of Virginia, \\ Charlottesville, Virginia; ${ }^{4}$ Department of Pathology, University of Virginia, Charlottesville, Virginia; ${ }^{5}$ The Cancer Center, University of \\ Virginia, Charlottesville, Virginia; and ${ }^{6}$ Department of Public Health Sciences, University of Virginia, Charlottesville, Virginia
}

The inability to successfully treat women with ovarian cancer is due in large part to the advanced stage of disease at diagnosis, the development of platinum resistance, and the lack of sensitive methods to monitor tumor progression and response to treatment. Vascular cell adhesion molecule-1 (VCAM-1) is expressed on the mesothelium of ovarian cancer patients. We investigated VCAM-1 expression as a marker of peritoneal metastasis and tumor response to platinum-based chemotherapy. Methods: Peritoneal or omental biopsies obtained from women diagnosed with stage I, stage II, or stage III/IV ovarian cancer were evaluated by immunohistochemistry. The effects of carboplatin on mesothelial VCAM-1 expression were determined in cultured cells by Western blot. Radiolabeled VCAM-1-specific peptide imaging probes and SPECT were used in a mouse model of ovarian cancer peritoneal metastasis to identify VCAM-1 as a viable imaging target. Results: VCAM-1 expression correlated with tumor stage. All specimens from stage I patients were negative, whereas $29 \%$ of stage II patients and $73 \%$ of stage III/IV patients were positive. Although most women with advanced stage disease expressed VCAM-1, the incidence of expression was reduced among women who received neoadjuvant chemotherapy, suggesting a role for chemotherapy in regulating VCAM-1 expression. Treatment of mesothelial cells in culture with carboplatin resulted in a transient decrease in VCAM-1 expression $4 \mathrm{~h}$ after treatment that returned to baseline within 16-24 $\mathrm{h}$. In vivo imaging of VCAM-1 also demonstrated an acute decrease in expression $4 \mathrm{~h}$ after carboplatin administration that recovered within $48 \mathrm{~h}$ in mice harboring platinum-resistant tumors. Chronic VCAM-1 expression reflected the effect of platinum-based treatment on tumor burden. Specifically, carboplatin treatment of mice with platinum-sensitive tumors showed reduced VCAM-1 expression, which correlated with reduced tumor burden; mice with platinum-resistant tumors retained elevated VCAM-1 expression and tumor burden after treatment. Conclusion: Clinically relevant VCAM-1-specific imaging probes identify VCAM-1 expression as an indicator of ovarian cancer peritoneal metastasis and therapeutic response to platinum-based agents. These observations support testing the utility of VCAM-1 imaging probes to monitor treatment response in ovarian cancer patients, thus providing the potential to improve management of women with this disease.

Received Nov. 29, 2012; revision accepted May 14, 2013.

For correspondence or reprints contact: Jill K. Slack-Davis, Department of Microbiology, Immunology and Cancer Biology, University of Virginia Health Sciences System, P.O. Box 800734, Charlottesville, VA 22908.

E-mail: jks6a@virginia.edu

Published online Sep. 12, 2013.

COPYRIGHT (C) 2013 by the Society of Nuclear Medicine and Molecular Imaging, Inc.
Key Words: VCAM-1; ovarian cancer; metastasis; SPECT/CT imaging

J Nucl Med 2013; 54:1883-1889

DOI: 10.2967/jnumed.112.117796

$\mathbf{O}$ varian cancer is the most lethal gynecologic malignancy, with an overall $5-y$ survival of $30 \%-40 \%$ (1). This statistic is somewhat misleading because women with early stage ovarian cancer account for about $30 \%$ of diagnoses and enjoy a 5-y survival of more than $70 \%$; however, $70 \%$ of ovarian cancer patients are diagnosed with advanced stage metastatic disease and experience a 5-y survival of less than $30 \%$. Stage III ovarian cancer, characterized by extensive spread throughout the peritoneal cavity, is diagnosed in $55 \%$ of patients, and stage IV—which includes the extensive peritoneal spread that identifies stage III disease as well as hematogenous metastasis to distant organs including lung and liver-occurs in $15 \%$ of patients.

The unfavorable prognosis for women with advanced stage ovarian cancer is due in large part to the existence or development of platinum-resistant disease. The current standard of care in the United States for women with advanced ovarian cancer is upfront surgical cytoreduction followed by systemic platinum/taxanebased chemotherapy (2). Although extensive retrospective data support aggressive surgery as important in improving overall survival (3), some will present with disease that is not resectable (e.g., intraparenchymal liver metastases) or will be too medically ill to undergo surgery safely, without significant morbidity. In these cases, neoadjuvant chemotherapy has been shown to allow optimal cytoreduction without significant morbidity (4). Unfortunately, as many as $20 \%$ of women in either scenario (i.e., upfront surgery or neoadjuvant chemotherapy) will not achieve first remission, and some will experience disease progression during primary therapy. Up to $90 \%$ of women who entered remission will experience recurrence through a process that is poorly understood and succumb to platinum-resistant disease within $2-3$ y. Currently, it is not possible to identify patients preoperatively who will respond to primary therapy. Moreover, patients with platinum-resistant disease are identified only after failure to respond to therapy; the definition of platinum sensitivity is based largely on retrospective data.

Presently, CA125, the only marker for ovarian cancer, is approved for use to assess treatment response. However, levels of CA125 are not predictive of successful cytoreduction. More- 
over, some patients will experience recurrence without a rise in CA125, and recent data suggest that rising CA125 in the setting of recurrent disease is not useful in identifying the appropriate time to start treatment (5). Finally, it has been noted that the traditional disease response to treatment as measured by CT scan and CA125 may not apply to the newer biologic therapies (6). There is therefore a need to identify new markers and to develop technologies to guide treatment decisions at different phases of this disease (7).

We identified vascular cell adhesion molecule-1 (VCAM-1) as a regulator of ovarian cancer peritoneal metastasis. Importantly, VCAM-1 is expressed preferentially on the mesothelium of ovarian cancer patients, compared with women with benign disease (8). Furthermore, inhibition of VCAM-1 function decreases mesothelial invasion, diminishes tumor burden, and increases survival in a mouse model of metastatic ovarian cancer (8). Therefore, we tested the hypothesis that VCAM-1 is a marker of peritoneal metastasis and tumor response to platinum-based chemotherapy. Moreover, we used a VCAM-1-targeted SPECT-based contrast agent to provide proof-of-principle data to support the clinical use of VCAM-1 imaging probes to monitor women treated for ovarian cancer.

\section{MATERIALS AND METHODS}

\section{Patients}

A retrospective review of patient data was performed with approval from the Institutional Review Board and met the criteria for waiver of consent under Code of Federal Regulations, sections 45CFR46.116 and 45CFR164.512(i)(2). The medical records of 58 epithelial ovarian cancer patients undergoing treatment at the University of Virginia (UVA) between 1998 and 2010 were retrospectively reviewed and clinicopathologic data including stage at diagnosis, grade, histology, treatment regimen, and degree of surgical cytoreduction obtained (Table 1). Among the 58 patients identified, 2 were excluded because of incomplete medical records and 9 because of inadequate tissue or staining.

With additional Institutional Review Board approval, a separate set of 32 patients with advanced stage disease based on treatment modality (i.e., neoadjuvant chemotherapy or primary surgical cyto- reduction) was identified; 1 patient was excluded because of inadequate cellular material with equivocal staining.

\section{VCAM-1 Immunohistochemistry}

Archived paraffin tissue blocks corresponding to each patient were obtained, sectioned, and stained for VCAM-1 by the Biorepository Tissue Research Facility at UVA using human-specific anti-VCAM-1 antibodies (Abcam) according to the manufacturer's instruction. Expression was scored as positive $(>50 \%)$ or negative $(<50 \%)$ with $3+$ intensity. Immunohistochemical staining of mouse tissue was performed by the Pathology Core at the UVA using anti-VCAM antibody specific for mouse (SC-1504; Santa Cruz) at a concentration of $5 \mu \mathrm{g} / \mathrm{mL}$.

\section{Cell Lines}

SKOV3ip1 cells, a platinum-resistant human ovarian cancer cell line, were grown in RPMI1640 supplemented with $15 \%$ fetal bovine serum (FBS). A2780 (platinum-sensitive) and A2780Cis (platinumresistant) ovarian cancer cells were grown in RPMI1640 supplemented with 10\% FBS and $2 \mathrm{mM} \mathrm{L}$-glutamine and for the A2780Cis, $1 \mu \mathrm{M}$ cisplatin every 2-3 passages. LP9 mesothelial cells (Coriell Repository) were grown in 1:1 medium 199 in Earle balanced salt solution: MCDB110 supplemented with 15\% FBS, 2 mM L-glutamine, epidermal growth factor $(10 \mathrm{ng} / \mathrm{mL})$, and hydrocortisone $(0.4 \mu \mathrm{g} / \mathrm{mL})$.

\section{Western Blot Analysis}

LP9 cells treated with increasing concentrations of carboplatin (UVA pharmacy) up to $1 \mu \mathrm{g} / \mathrm{mL}$ for $24 \mathrm{~h}$ or with $1 \mu \mathrm{g} / \mathrm{mL}$ for increasing periods of time up to $72 \mathrm{~h}$ were lysed in supplemented radioimmunoprecipitation assay buffer ( $50 \mathrm{mM}$ Hepes, $0.15 \mathrm{M} \mathrm{NaCl}, 2 \mathrm{mM}$ ethylenediaminetetraacetic acid, $0.1 \%$ Nonidet $\mathrm{P} 40$, and $0.05 \%$ sodium deoxycholate, $\mathrm{pH}$ 7.2) containing protease inhibitors (Roche protease cocktail) and $1 \mathrm{mM} \mathrm{Na}_{3} \mathrm{VO}_{4}, 40 \mathrm{mM} \mathrm{NaF}$, and $10 \mathrm{mM} \mathrm{Na}_{4} \mathrm{P}_{2} \mathrm{O}_{7}$. Proteins (10-25 $\mu \mathrm{g}$ of lysate) were resolved on $8 \%$ sodium dodecyl sulfate polyacrylamide gel electrophoresis gels, transferred to nitrocellulose, and blotted with antihuman VCAM-1 or anti-actin antibodies, followed by incubation with horseradish peroxidase-conjugated secondary antibodies and visualization by enhanced chemiluminescence (Amersham Biosciences). Films were scanned and VCAM-1 signal intensity determined using ImageJ (rsbweb.nih.gov) and normalizing the VCAM-1 signal to actin for each sample.

TABLE 1

Patient Demographic Data

\begin{tabular}{|c|c|c|c|}
\hline Patient characteristic & Stage $1 / I I$ & Stage III/IV & $P$ \\
\hline Number & 26 & 30 & - \\
\hline Median age $(y)$ & 56 & 60 & Not significant \\
\hline \multicolumn{4}{|l|}{ Histology } \\
\hline Serous & $10(38)$ & $25(83)$ & $<0.001$ \\
\hline Endometrioid & $7(27)$ & $1(3)$ & \\
\hline Mucinous & $2(8)$ & $1(3)$ & \\
\hline Clear cell & $4(15)$ & $2(7)$ & \\
\hline Mixed & $3(12)$ & - & \\
\hline Carcinosarcoma & - & $1(3)$ & \\
\hline Other & - & - & \\
\hline \multicolumn{4}{|l|}{ Treatment approach } \\
\hline Primary surgery & $25(96)$ & $26(87)$ & - \\
\hline Neoadjuvant chemotherapy & $1(4)$ & $4(13)$ & \\
\hline \multicolumn{4}{|l|}{ Surgical cytoreduction } \\
\hline Optimal & $26(100)$ & $18(60)$ & - \\
\hline Suboptimal & $0(0)$ & $9(30)$ & \\
\hline
\end{tabular}

Data in parentheses are percentages. 


\section{Quantitative Real-Time Polymerase Chain Reaction (PCR)}

RNA was extracted from treated LP9 cells using TRIzol reagent (Invitrogen), and complementary DNA was generated with an iScript cDNA synthesis kit (Bio-Rad). Quantitative real-time PCR was performed using the Step One Plus Real-Time PCR system (Applied Biosystems); the following primers specific for human VCAM-1: forward, CTTAAAATGCCTGGGAAGATGGT, and reverse, GTCAATGAGACGGAGTCACCAAT, or glucuronidase $\beta$-(GUSB; internal PCR control): forward, CCGACTTCTCTGACAACCGAC, and reverse, AGCCGACAAAATGCCGCAGACG; and Absolute Blue Syber Green ROX mix (Thermo Scientific). Data were analyzed using the $\Delta-\Delta \mathrm{C}_{\mathrm{T}}$ method.

\section{VCAM-1 Peptide}

The synthesis of a SPECT imaging agent with specificity for VCAM-1 has been previously described (9-11). A tetrameric VCAM-1-targeted peptide (tVCAM-4 ([(VHPKQHRGGSPEG5K)4K]2-KK(DOTA)$\left.\beta A-\mathrm{NH}_{2}\right)$ ) (11) was synthesized (CS Bio Co.) for SPECT/CT imaging by chelating radioactive indium to the DOTA. For indium labeling, the peptide $(100 \mu \mathrm{g})$ was dissolved in $20 \mu \mathrm{L}$ of phosphate-buffered saline (PBS) and then diluted in $300 \mu \mathrm{L}$ of ammonium acetate buffer $(0.1 \mathrm{M}$, $\mathrm{pH}$ 5.5). Indium chloride $(185 \mathrm{MBq}[5 \mathrm{mCi}]$ in water; Cardinal Health) was mixed with the peptide and allowed to equilibrate with mixing at $40^{\circ} \mathrm{C}$ for $10 \mathrm{~min}$.

\section{Animal Models}

All mouse experiments were performed in accordance with the policies and procedures established by the UVA Animal Care and Use Committee. NCR nude mice (Taconic Laboratories) were injected intraperitoneally with $10^{6}$ SKOV3ip1 (platinum-resistant; kindly provided by Dr. Anil Sood, M.D. Anderson), A2780 (platinum-sensitive; Sigma-Aldrich, Inc.), or A2780Cis (platinum-resistant; Sigma-Aldrich, Inc.) cells suspended in $200 \mu \mathrm{L}$ of PBS as previously described (8). Control nude mice received a single intraperitoneal injection of 200 $\mu \mathrm{L}$ of PBS. Two, 3, and $4 \mathrm{wk}$ after SKOV3ip1 cell injection $(n=3$ per group), mice were imaged and euthanized, and biodistribution of the labeled tetrameric synthetic peptide was assessed. To evaluate VCAM-1 as a marker of chemosensitivity, a cohort of mice $(n=9)$ was injected with SKOV3ip1 cells. Groups of 3 mice were imaged 2 wk after tumor initiation, $8 \mathrm{~h}$ after a single intraperitoneal injection of carboplatin ( $25 \mathrm{mg} / \mathrm{kg}$; administered $2 \mathrm{wk}$ after tumor initiation), or $2 \mathrm{~d}$ after carboplatin injection. After each imaging session, mice were euthanized, and probe biodistribution was determined. To evaluate the effects of longterm exposure to chemotherapy on VCAM-1 expression, one group of mice $(n=9)$ was injected with A2780 (platinum-sensitive cell line) and another $(n=9)$ with A2780Cis (platinum-resistant cell line). Mice were treated with carboplatin $(25 \mathrm{mg} / \mathrm{kg})$ beginning $1 \mathrm{wk}$ after tumor initiation and continuing weekly thereafter. Cohorts of 3 mice per cell line were imaged $1 \mathrm{wk}$ after tumor initiation, just before treatment (week 0 ), and then 1 and 2 wk after initiation of treatment, and ${ }^{111}$ In- tVCAM-4 uptake was quantified via biodistribution.

\section{Peptide Chelation}

For indium labeling, the peptide $(100 \mu \mathrm{g})$ was dissolved in $10 \mu \mathrm{L}$ of PBS, then for every $185 \mathrm{MBq}(5 \mathrm{mCi})$ of indium, $5 \mu \mathrm{L}$ were removed and added to an Eppendorf tube containing $500 \mu \mathrm{L}$ of ammonium acetate buffer ( $0.1 \mathrm{M}, \mathrm{pH} 5.5)$. Indium chloride was added to the tub containing the peptide-ammonium acetate buffer and mixed in a Thermomixer (Eppendorf) $\left(1,000 \mathrm{RPM}, 40^{\circ} \mathrm{C}\right)$ for $10 \mathrm{~min}$. After being mixed, the chelated peptide was removed and assessed for radiochemical purity using a Sep-Pak cartridge (Waters Corp.) to pass a radiochemical purity of $90 \%$ or more.

\section{Imaging}

Mice were injected with $37 \mathrm{MBq}(1 \mathrm{mCi})$ of ${ }^{111} \mathrm{In}$-labeled tVCAM-4 (11) and then imaged $4 \mathrm{~h}$ after injection with a micro-SPECT/CT scanner designed and built at UVA. CT acquisition used 200 evenly spaced projections spanning $200^{\circ}$ over approximately $5 \mathrm{~min}$. Pinhole SPECT scanning was then performed using 2 opposing $\gamma$-cameras simultaneously. The 2 cameras were fitted with 0.5 -mm-diameter tungsten pinholes. Sixty evenly spaced projection views per camera were obtained over $180^{\circ}$, for a total of 120 views at $3^{\circ}$ increments over $360^{\circ}$. The SPECT acquisition time was approximately $45 \mathrm{~min}$. The reconstructed CT voxel size was $0.082 \times 0.082 \times 0.082 \mathrm{~mm}$ on a $640 \times 640 \times 768$ image matrix. The reconstructed SPECT voxel size was $0.65 \times 0.65 \times 0.65 \mathrm{~mm}$ on an $80 \times 80 \times 80$ image matrix. All SPECT images were corrected for radioactivity decay but not for $\gamma$-ray attenuation.

\section{Biodistribution}

After mice were imaged via SPECT/CT, animals were sacrificed and their organs harvested and placed into preweighed Eppendorf tubes. Each tube was then reweighed to determine the weight of the organ and the radiation measured using a $\gamma$-counter. Data represent the mean \pm SD and are presented as dose injected per gram of tissue.

\section{Statistical Analysis}

The significance of mesothelial VCAM-1 expression in patients was analyzed using $\chi^{2}$ and Fisher exact tests. Animal experiments were analyzed using ANOVA followed by Bonferroni adjustment for all combinations.

\section{RESULTS}

VCAM-1 is expressed preferentially on the mesothelium of ovarian cancer patients, compared with women with benign disease (8), and inhibition of VCAM-1 function decreases mesothelial invasion, diminishes tumor burden, and increases survival in a mouse model of metastatic ovarian cancer (8). Because mesothelial VCAM-1 regulates ovarian cancer metastasis, we examined the relationship between expression and tumor progression in an attempt to determine whether VCAM-1 would function as a marker of progression. Therefore, the association between peritoneal VCAM-1 expression and clinical parameters of disease including tumor stage, optimal cytoreduction, time to recurrence, and overall outcome was investigated (Table 1). There was no significant difference in age or treatment approach between early (stage I/II) and late (stage III/IV) stage disease. As expected, most of the late stage patients were diagnosed with serous papillary histologic subtype, and a greater proportion of them were suboptimally cytoreduced (Table 1 ).

Biopsies of the peritoneum or omentum were examined for mesothelial VCAM-1 expression after immunohistochemistry staining (Fig. 1A). The incidence of VCAM-1 expression increased with tumor stage. None of the women diagnosed with stage I ovarian cancer expressed VCAM-1, whereas VCAM-1 was detected in 4 of 14 (29\%) stage II patients and 16 of 22 (73\%) patients with advanced stage (III/IV) disease (Fig. 1B). To understand the discrepancies in VCAM-1 expression among stage II or stage III/IV patients, additional clinical and pathologic features were reviewed. Stage II ovarian cancer is defined as spread within the pelvis (2). This definition includes the development of large tumors encompassing the primary site and adjacent tissues or the establishment of secondary tumor implants within the pelvis. Among the stage II patients, 7 had secondary implants, and 3 of those showed VCAM-1 expression; only 1 patient with adnexa-only disease showed VCAM-1 positivity (Fig. 1C). Similarly, most women with stage III/IV disease, which is characterized by extensive implantation of tumors within the entire 


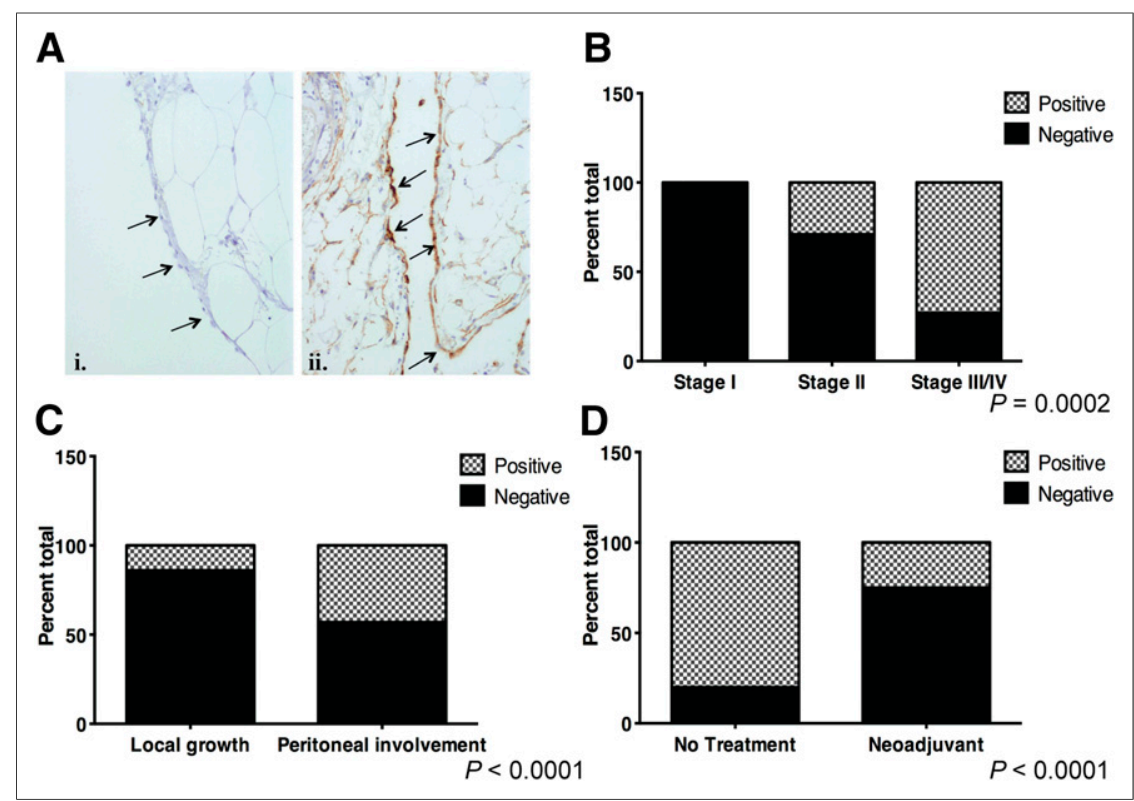

FIGURE 1. Peritoneal VCAM-1 expression among women with ovarian cancer. (A) Representative histology of biopsies stained for VCAM-1 expression: i-example of negative staining; iibrown staining of mesothelium indicates positive reactivity. Arrows indicate mesothelium. (B) Incidence of women with positive VCAM-1 staining of mesothelium segregated by tumor stage. Omentum or peritoneal biopsies were stained for VCAM-1 using immunohistochemistry. Specimens were scored positive if more than $50 \%$ of mesothelial cells showed $3+$ reactivity (stippled bars) or negative if less than $50 \%$ of cells showed reactivity (solid gray). Data represent percentage of total patients analyzed $\left(P=0.0002, \chi^{2}\right.$ test). Stage I, $n=12$; stage II, $n=14$; stage III/IV, $n=22$. (C) Percentage of specimens with VCAM-1 positivity among stage II patients separated on basis of presence (peritoneal involvement, $n=7$ ) or absence (local growth, $n=7$ ) of secondary tumor implants within pelvis $(P<0.0001$, Fisher exact test). (D) Percentage of VCAM-1 staining specimens from set of matched stage III patients who received upfront surgery (no treatment, $n=18)$ or neoadjuvant chemotherapy $(n=13)(P<$ 0.0001 , Fisher exact test).

peritoneal cavity, expressed VCAM-1 (16/22, Fig. 1B). These findings indicate that VCAM-1 expression is associated with peritoneal metastasis, and expression is evident at the earliest indication of secondary tumor formation.

Among the patients with stage III/IV ovarian cancer, 6 of the 22 stained specimens lacked VCAM-1 reactivity. Evaluation of the clinical data from the stage III/IV patients indicated that $18 \%$ (4/ 22) received neoadjuvant chemotherapy (Table 1), an option provided to women with excessive tumor burden in an attempt to optimize the chances of successful cytoreductive surgery. Interestingly, VCAM-1 staining was observed in just 1 patient who received neoadjuvant chemotherapy (data not shown). To more thoroughly evaluate the possibility that neoadjuvant chemotherapy alters VCAM-1 expression, a second set of patients-including 18 undergoing primary cytoreductive surgery and 13 undergoing interval cytoreduction after neoadjuvant chemotherapy-was evaluated. Analysis revealed significantly fewer women with mesothelial VCAM-1 expression after exposure to neoadjuvant chemotherapy, as compared with primary surgery $(15 \%$ [2/13] vs. $72 \%$ [13/18], respectively) (Fig. 1D). Together, these observations indicate that in addition to the presence of VCAM- 1 at the earliest stages of peritoneal spread (i.e., stage II patients with secondary implants), expression is responsive to treatment. This observation implies that VCAM-1 is regulated directly by platinum agents. Alternatively, because neoadjuvant chemotherapy is provided to reduce tumor burden, its effect on VCAM-1 expression might not be direct but rather a result of the loss of tumor, thus offering the tantalizing hypothesis that VCAM-1 could be used to reflect tumor burden and identify patients responding to platinum-based treatment.

These possibilities were tested directly in a mouse model of peritoneal ovarian cancer metastasis using SPECT/CT with a VCAM-1-targeted ${ }^{111}$ In-labeled imaging agent ( ${ }^{111}$ In-tVCAM-4) (9-13). Previous work has demonstrated the specificity and high affinity of tVCAM-4 for VCAM-1 (11). Similar to the reported pharmacokinetic data for this probe, we observed the highest accumulation of probe in the kidney $4 \mathrm{~h}$ after injection (Supplemental Table; supplemental materials are available at http://jnm.snmjournals.org). Using immunohistochemistry, we previously reported that VCAM-1 is expressed on the mesothelium of tumor-bearing mice within 2 wk of tumor initiation, a time when tumors are microscopic (8). Therefore, mice were injected intraperitoneally with SKOV3ip1 cells and imaged 2 wk later using ${ }^{111} \mathrm{In}$ tVCAM-4 and SPECT/CT (Fig. 2; Supplemental Fig. 1). Imaging of tumor-bearing mice demonstrated diffuse intraperitoneal labeling in regions consistent with the omentum and mesentery (Fig. 2A), primary sites of metastasis in the mouse model. In contrast, mice without tumors had kidney uptake (Fig. 2A, left) consistent with kidneys being the primary route of clearance $(10,11)$. Correlative histology demonstrated the presence of VCAM-1 on the mesothelium of tumor-bearing mice (Fig. 2B). Confirmation of imaging was provided through biodistribution analysis of omentum, mesentery, and associated tumors and uninvolved organs such as the heart. Omentum and mesentery from tumor-bearing animals had a 10fold-higher accumulation of the VCAM-1-targeted agent than omentum and mesentery from control animals (Figs. 2C). To determine the extent and duration of expression, mice were evaluated weekly after tumor initiation starting at $2 \mathrm{wk}$. As indicated by biodistribution, maximal VCAM-1 expression was detected $2 \mathrm{wk}$ after tumor initiation and remained elevated through the course of the experiment (4 wk total, Fig. 2C). With the exception of a slight, statistically significant, increase $3 \mathrm{wk}$ after tumor initiation, levels of VCAM-1 probe uptake in the heart were unaffected between tumor-bearing animals and control animals. Together, these observations validate the use of radiolabeled imaging probes to monitor VCAM-1 expression in a mouse model of peritoneal ovarian cancer metastasis and demonstrate that VCAM- 1 is expressed early in the course of disease. However, expression does not mirror the kinetics of tumor growth; maximal VCAM-1 expression was observed with microscopic tumors and did not change as tumor burden continued to increase (data not shown).

The retrospective patient data demonstrated a lower incidence of VCAM-1 expression among patients exposed to neoadjuvant chemotherapy. We considered 2 possibilities to account for this observation. First, mesothelial VCAM-1 expression is targeted 
directly by platinum agents, or second, metastatic ovarian cancer creates an environment that stimulates and maintains VCAM-1 expression in vivo, implying that expression reflects tumor responsiveness to chemotherapy. To determine whether carboplatin affects VCAM-1 expression directly, LP9 mesothelial cells, a primary human culture that constitutively expresses VCAM-1 and was isolated from a patient with ovarian cancer (14), were treated with increasing concentrations of carboplatin, a standard chemotherapy for the treatment of ovarian cancer. None of the concentrations tested altered VCAM-1 expression (Fig. 3A) or cell viability (data not shown). However, patients are unlikely to be exposed to carboplatin continuously for $24 \mathrm{~h}$ or more. Indeed, up to $80 \%$ of the injected dose is excreted within $24 \mathrm{~h}$ (15). In an attempt to approach the in vivo cellular exposure, LP9 cells were pulsed with carboplatin $(1 \mu \mathrm{g} / \mathrm{mL})$ for $1 \mathrm{~h}$ and allowed to recover in fresh medium for the indicated times (Fig. 3B; Supplemental Fig. 2). Within $1 \mathrm{~h}$ after carboplatin exposure, VCAM-1 messenger RNA and protein levels decreased dramatically before returning to baseline levels 8-16 h after exposure (Fig. 3B; Supplemental Fig. 2 ), where they remained elevated for up to $72 \mathrm{~h}$ (data not shown). These observations indicate that carboplatin induces a transient decrease in VCAM-1 expression in cultured human mesothelial cells.

Although treatment with carboplatin generates a direct albeit transient decrease in VCAM-1 expression, the mesothelial cell line examined constitutively expresses VCAM-1, which is not observed

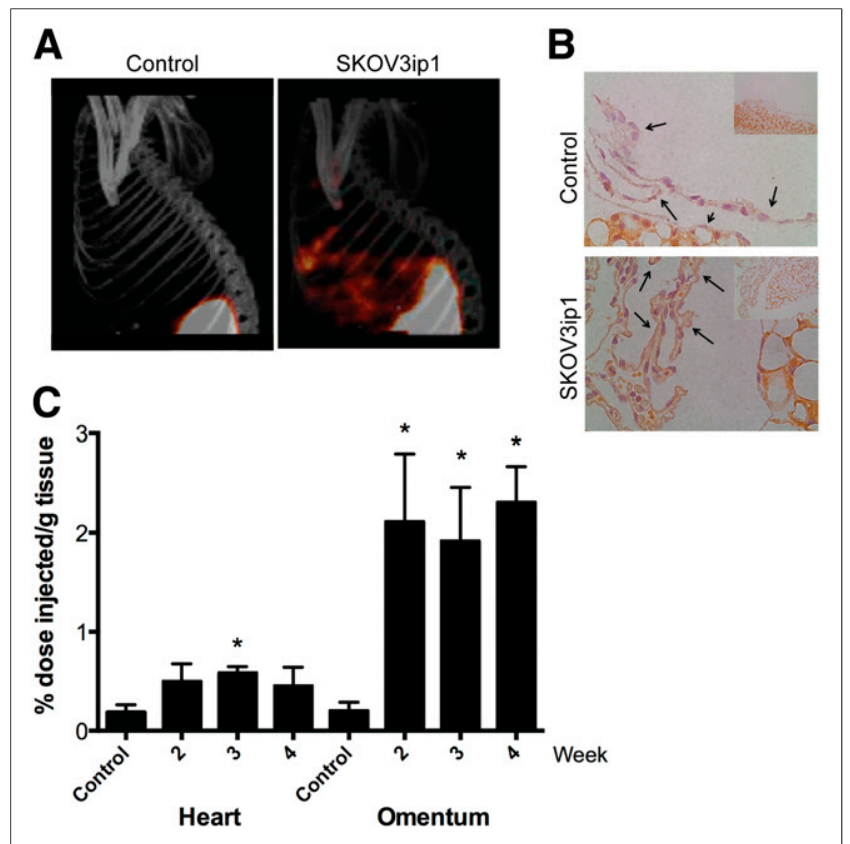

FIGURE 2. Detection of mesothelial VCAM-1 expression in mouse model of ovarian cancer peritoneal metastasis. Athymic nude mice received intraperitoneal injection of SKOV3ip1 cells or saline (control). (A) SPECT/CT imaging of ${ }^{111} \mathrm{In}$-tVCAM- 4 peptide $4 \mathrm{~h}$ after intraperitoneal injection into saline control-injected or tumor-bearing animals. Maximum-intensity-projection images are shown. (B) Correlative histology. After radioactive decay, omentums were stained for VCAM-1 using immunohistochemistry (60x images with $20 \times$ insert). Arrows indicate mesothelium in each case. (C) Kinetics of VCAM-1-111 In peptide distribution in heart or omentum of athymic nude mice 2, 3, and 4 wk after intraperitoneal injection of SKOV3ip1 cells, $n=3$. Control animals were evaluated $2 \mathrm{wk}$ after PBS injection. Data represent mean \pm SD. $P<0.05,1$-way ANOVA for heart or omentum samples. ${ }^{\star} P<0.05$, Bonferroni multiplecomparison test, relative to PBS-injected control for each organ. in healthy women. Rather, VCAM-1 expression is induced in women with ovarian cancer peritoneal metastases (Fig. 1). Moreover, the tumor microenvironment is much more complex than that achieved in cell culture. To determine whether carboplatin alters ovarian cancer-induced VCAM-1 expression in vivo, mice harboring platinum-resistant (SKOV3ip1 or A2780Cis) or platinumsensitive (A2780) ovarian cancer cells were evaluated. First, the short-term effects of exposure to carboplatin on mesothelial VCAM-1 expression were determined. Two weeks after intraperitoneal injection of SKOV3ip1 cells, mice $(n=3)$ were imaged using the VCAM-1-targeted imaging agent to obtain baseline expression levels. Two days later, mice $(n=6)$ were treated with carboplatin for $4 \mathrm{~h}$; 3 of the treated mice were injected with the VCAM-1 imaging agent and imaged $4 \mathrm{~h}$ later. The remaining 3 mice were imaged $2 \mathrm{~d}$ after carboplatin administration. Treatment with carboplatin resulted in a slight decrease in biodistribution of ${ }^{111}$ In-tVCAM4 to the omentum; however, this change was not statistically significant (Fig. 3C). Within $2 \mathrm{~d}$ of treatment, VCAM-1 expression not only recovered to baseline, it was $50 \%$ greater. This observation demonstrates that carboplatin does not alter VCAM-1 expression in vivo in the presence of platinum-resistant tumors. Moreover, VCAM-1 expression increases above baseline within $2 \mathrm{~d}$, thus raising the possibility that ovarian cancer-dependent VCAM-1 expression in vivo is maintained by the continued presence of the tumor.

To test this hypothesis, platinum-sensitive (A2780) or -resistant (A2780Cis) ovarian cancer cells were used to generate peritoneal tumors that were then treated with carboplatin and imaged. Baseline VCAM-1 expression (before carboplatin treatment) was determined using SPECT/CT $1 \mathrm{wk}$ after tumor initiation with each cell line (Fig. 4A; Supplemental Fig. 3, week 0). Each group of mice was treated with $25 \mathrm{mg}$ of carboplatin per kilogram weekly and imaged 1 and 2 wk after initial treatment just before subsequent weekly dosing. As shown in Figure 4 and Supplemental Figure 3, VCAM-1 expression was detected 1 wk after tumor initiation in both models (week 0). Tumors in mice containing A2780Cis cells continued to grow in the presence of carboplatin (Fig. 4C). Concomitantly, VCAM-1 expression in this model increased within 2 wk of tumor initiation, demonstrating a 2.5 -fold increase in uptake of ${ }^{111}$ In-tVCAM-4 peptide after $2 \mathrm{wk}$ of carboplatin treatment (Figs. 4A and 4B; Supplemental Fig. 3). In contrast, omentum from animals with tumors generated from platinum-sensitive A2780 cells had lower, although not statistically significant, levels of ${ }^{111}$ In-tVCAM-4 uptake after 2 wk of treatment (Figs. 4A and 4B; Supplemental Fig. 3). These observations indicate that VCAM-1 expression kinetics can be monitored in vivo and that carboplatin treatment does not diminish VCAM-1 expression in mice harboring platinum-resistant tumors; however, expression is curbed when tumor growth is inhibited by platinum treatment.

\section{DISCUSSION}

This study demonstrates a potential role for VCAM-1 as a marker of ovarian cancer peritoneal metastasis and tumor responsiveness to platinum-based chemotherapy. Retrospective analysis of omental or peritoneal biopsies revealed an increased incidence of mesothelial VCAM-1 expression with increasing tumor stage such that expression was coincident with secondary tumor implantation. Additionally, the incidence of VCAM-1 expression was reduced among women who received neoadjuvant chemotherapy to reduce tumor burden before surgery. Using clinically relevant VCAM-1-targeted imaging probes and a mouse model of peritoneal 


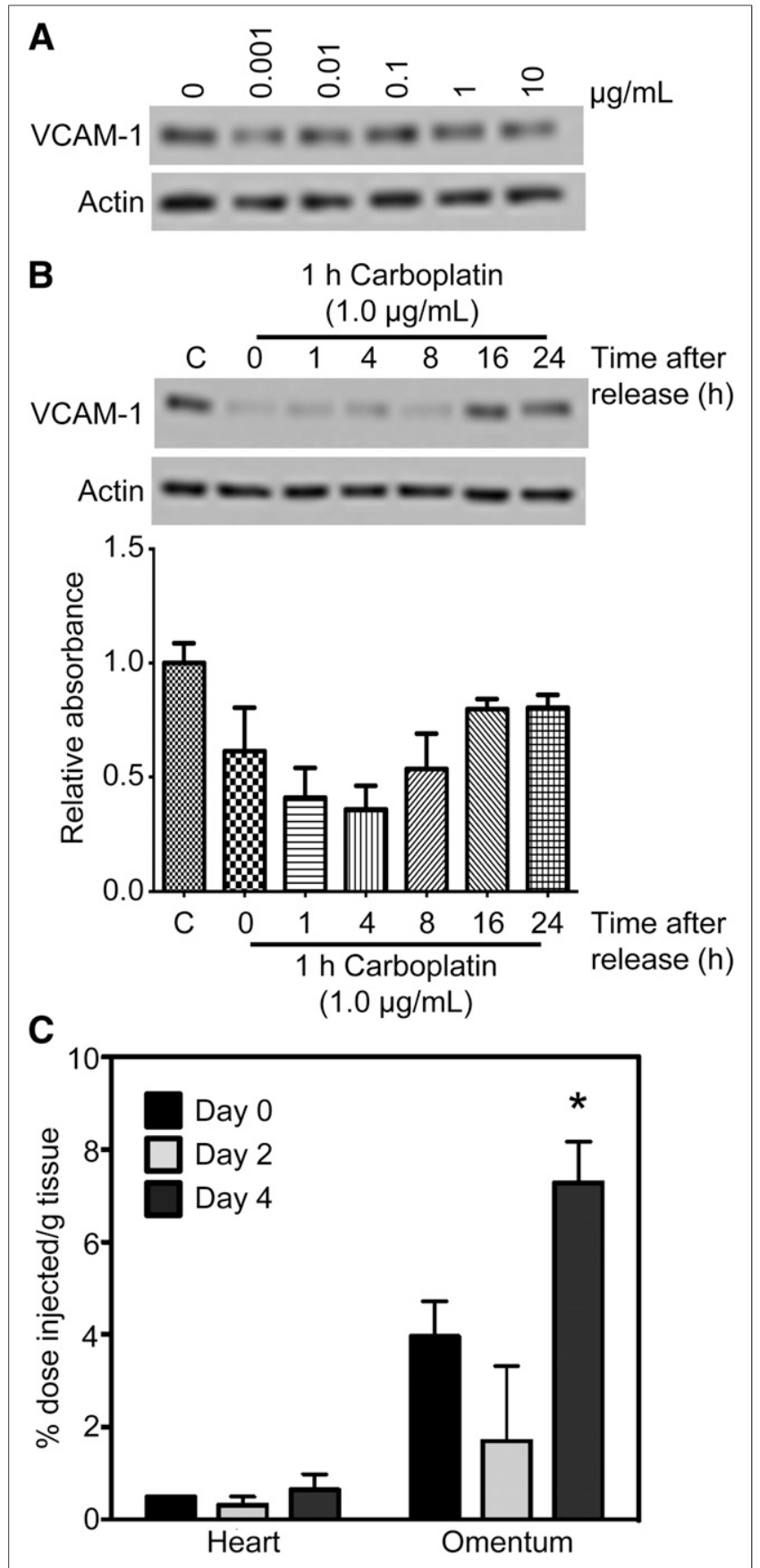

FIGURE 3. Carboplatin transiently diminishes mesothelial VCAM-1 expression. (A) LP9 mesothelial cells were treated with indicated concentrations of carboplatin for $24 \mathrm{~h}$ before lysis and immunoblotting for VCAM-1 and actin (loading control). (B) LP9 cells were either untreated or exposed to carboplatin $(1 \mu \mathrm{g} / \mathrm{mL})$ for $1 \mathrm{~h}$ before release into fresh medium. Lysates were blotted for VCAM-1 and actin. After background subtraction, signal intensities for VCAM-1 bands were normalized to actin and are expressed relative to untreated control (designated by C). (C) Nude mice harboring SKOV3ip1 tumors were imaged using SPECT/CT and ${ }^{111} \mathrm{In}$-tVCAM-4 peptide 2 wk after tumor initiation to obtain baseline distribution values (day 0 ). Two days later (day 2), mice were imaged $8 \mathrm{~h}$ after receiving intraperitoneal injection of carboplatin (25 mg/kg) and after another $2 \mathrm{~d}$ (day 4); ${ }^{111} \mathrm{In}$-tVCAM-4 peptide biodistribution is presented as mean \pm SD. ovarian cancer metastasis, we detected maximal mesothelial VCAM-1 expression with microscopic tumor burden. Importantly, VCAM-1 expression mirrored tumor response to platinum-based chemotherapy. VCAM-1 expression was negligible in mice bearing platinum-sensitive ovarian cancer cells after treatment with carboplatin; expression continued to increase and was maintained in mice harboring platinum-resistant tumors after treatment. Together, these observations support a role for VCAM-1 as an indicator of peritoneal ovarian cancer metastasis and responsiveness to platinum-based chemotherapy and offer the potential for VCAM-1-targeted imaging agents to change ovarian cancer clinical management.

The observation that VCAM-1 is expressed on the mesothelium of ovarian cancer patients at the earliest stage of secondary implantation (i.e., stage II with secondary implants within the pelvis) raises the intriguing possibility that VCAM-1 could function as a marker of peritoneal metastasis. The ability to identify peritoneal metastasis early in the course of disease has implications for women at high risk for developing ovarian cancer or those with stage II disease where VCAM-1 expression might identify those at risk for progressing to stage III ovarian cancer. Current methodology to evaluate VCAM-1 expression in patients relies on nonquantitative immunohistochemistry, which is limited further by the number of biopsies taken from each patient. Using a VCAM1-specific imaging agent originally designed to monitor atherosclerosis (9-11), we observed maximal VCAM-1 expression in a mouse model of peritoneal ovarian cancer metastasis with microscopic tumor burden. Using more quantitative imaging modalities such as PET (11) will improve the ability to demonstrate VCAM-1 as a marker of peritoneal metastasis.

In addition to indicating early metastasis, the ability to detect maximal mesothelial VCAM-1 expression with microscopic tumor burden has implications for monitoring women for recurrence. After the initial successful treatment of metastatic ovarian cancer, which occurs for $80 \%$ of patients, most women will experience a recurrence within $2 \mathrm{y}$ and eventually succumb to their disease (2). Current modalities to monitor women who have entered remission are ineffective. Serum CA125 is not a reliable predictor of recurrence, CT scans are unable to detect small or diffuse lesions that are likely to affect prognosis, and neither modality predicts the appropriate time to treat recurrent disease (5-7). Thus, imaging VCAM-1 may provide a more reliable indication as to when to initiate second-line therapy.

Our data demonstrating reduced incidence of VCAM-1 expression among women who received neoadjuvant chemotherapy together with the observation that VCAM-1 expression mirrors tumor response to carboplatin treatment implicate mesothelial VCAM-1 expression as a marker of tumor response to platinumbased chemotherapy. Although carboplatin induced a direct, transient decrease in VCAM-1 expression in cultured mesothelial cells, the effect was lost in mice harboring platinum-resistant tumors. Importantly, VCAM-1 expression was able to differentiate platinum-sensitive tumors from -resistant ones within $1 \mathrm{wk}$ of treatment. The development of resistance to platinum-based chemotherapy is a major obstacle for the successful treatment of metastatic ovarian cancer. Unfortunately, to date this phenomenon is defined somewhat loosely based on retrospective data; patients are categorized as having platinum-resistant disease only after having failed primary therapy with persistent disease or progression within 6 mo of completion of therapy (16). The ability to objectively identify ovarian cancers that are resistant 


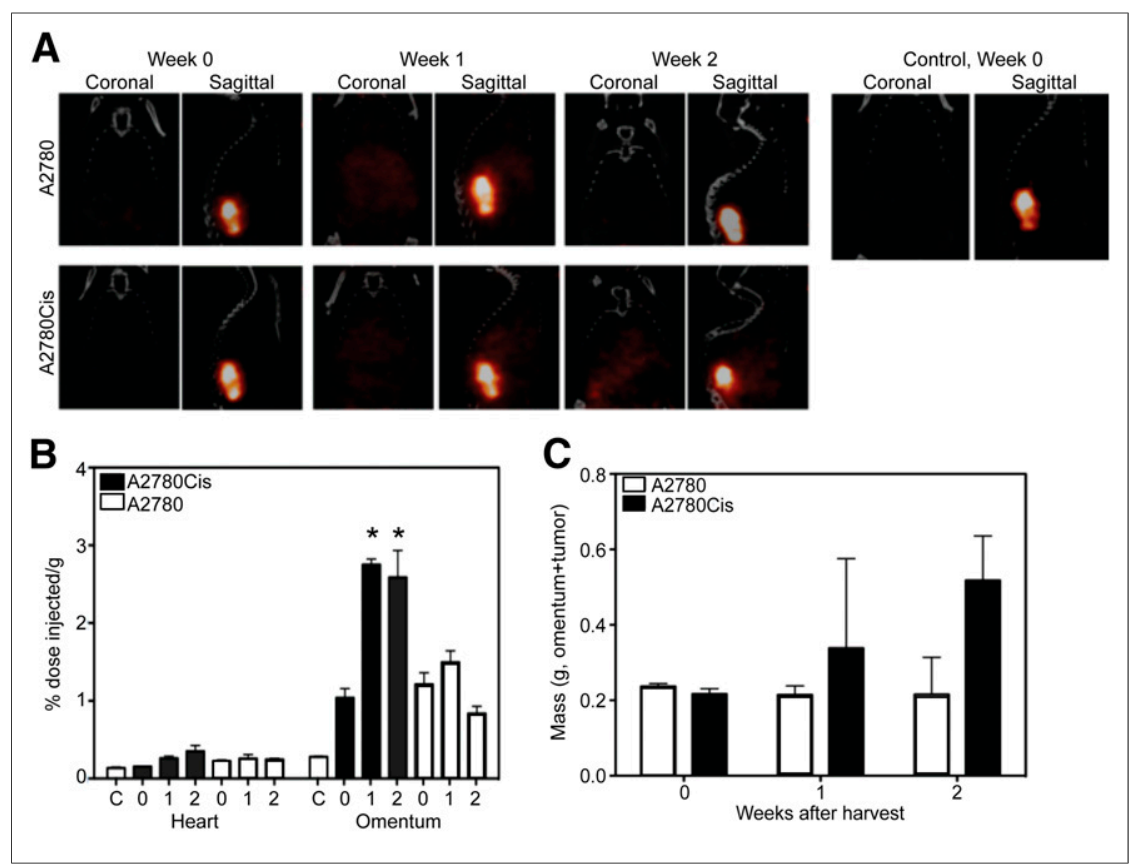

FIGURE 4. Carboplatin indirectly regulates chronic mesothelial VCAM-1 expression by affecting tumor burden. Nude mice were injected with A2780 ( $n=3$ per time point) or A2780Cis ( $n=3$ per time point) cells or saline (control, $n=2$ ). One week after tumor initiation, a group of mice was imaged to obtain baseline values; remaining mice were treated with carboplatin $(25 \mathrm{mg} / \mathrm{kg})$ weekly. (A) SPECT/CT images were collected weekly starting $1 \mathrm{wk}$ after tumor initiation (week 0 ); weeks 0,1 , and 2 indicate time after carboplatin treatment. Representative coronal and sagittal maximum-intensity-projection images of ${ }^{111} \mathrm{In}$-tVCAM- 4 peptide obtained $4 \mathrm{~h}$ after intraperitoneal injection are depicted. (B) Biodistribution of ${ }^{111} \mathrm{In}$-tVCAM-4. Heart and omentum were removed from each cohort of mice weekly after carboplatin treatment $(x$-axis, $0=1 \mathrm{wk}$ tumor, no treatment) and percentage injected dose of radioactivity per gram of tissue determined. $n=3$ for each cohort; $\mathrm{C}=$ control animals; 0,1 , and 2 indicate weeks after carboplatin treatment. $P<0.05,1$ way ANOVA for omentum samples; ${ }^{*} P<0.05$, Bonferroni multiple-comparison test, relative to week 0, A2780 or A2780Cis, which were not different from each other. (C) Omentum (primary site of tumor deposition) and any visible tumors from peritoneal cavity were removed and weighed. Data represent mean mass \pm SEM $(n=3)$. gray bars $=$ A2780-tumored mice; black bars $=$ A2780Cis-tumored mice.

versus sensitive to platinum agents would provide a significant advance in the management of women with metastatic ovarian cancer. Importantly, a molecular indication of chemosensitivity could allow for earlier detection of platinum-resistant disease to permit alterations in treatment regimen directed at the molecular behavior of disease. Moreover, it would potentially provide an opportunity to objectively identify women for inclusion in clinical trials and an ability to monitor their progress.

\section{CONCLUSION}

VCAM-1 may serve as a sensitive indicator of ovarian cancer peritoneal metastasis and response to platinum-based chemotherapy. Although additional studies are necessary to determine the prognostic implications of VCAM-1 expression among ovarian cancer patients, strategies designed to image mesothelial VCAM-1 expression in ovarian cancer patients offer the potential to improve management of women with this disease.

\section{DISCLOSURE}

The costs of publication of this article were defrayed in part by the payment of page charges. Therefore, and solely to indicate this fact, this article is hereby marked "advertisement" in accordance with 18 USC section 1734 . This study was supported by the Gynecologic Oncology Fellowship program, in part by a grant from the University of Virginia Women's Oncology Research Fund and Cancer Center, and by NCI R01 CA142783. No other potential conflict of interest relevant to this article was reported.

\section{ACKNOWLEDGMENTS}

We are grateful to the Molecular Imaging Core Facility, specifically Drs. Mark Williams and Kosta Popovic for their assistance with in vivo imaging; the Biorepository and Tissue Research Facility for assistance with obtaining and staining patient samples; and the Pathology Core for staining mouse tissues.

\section{REFERENCES}

1. Siegel R, Naishadham D, Jemal A. Cancer statistics, 2012. CA Cancer J Clin. 2012;62:10-29.

2. Ozols RF. Treatment goals in ovarian cancer. Int $J$ Gynecol Cancer. 2005;15(suppl 1):3-11.

3. Eisenhauer EL, Abu-Rustum NR, Sonoda Y, Aghajanian C, Barakat RR, Chi DS. The effect of maximal surgical cytoreduction on sensitivity to platinum-taxane chemotherapy and subsequent survival in patients with advanced ovarian cancer. Gynecol Oncol. 2008;108:276-281.

4. Hou JY, Kelly MG, Yu H, et al. Neoadjuvant chemotherapy lessens surgical morbidity in advanced ovarian cancer and leads to improved survival in stage IV disease. Gynecol Oncol. 2007;105: 211-217.

5. Rustin GJ, van der Burg ME, Griffin CL, et al. Early versus delayed treatment of relapsed ovarian cancer (MRC OV05/EORTC 55955): a randomised trial. Lancet. 2010;376:1155-1163.

6. Rustin GJ. Use of CA-125 to assess response to new agents in ovarian cancer trials. J Clin Oncol. 2003;21:187s-193s.

7. Eisenhauer EA. Optimal assessment of response in ovarian cancer. Ann Oncol. 2011;22(suppl 8):viii49-viii51.

8. Slack-Davis JK, Atkins KA, Harrer C, Hershey ED, Conaway M. Vascular cell adhesion molecule-1 is a regulator of ovarian cancer peritoneal metastasis. Cancer Res. 2009;69:1469-1476.

9. Kelly KA, Nahrendorf M, Yu AM, Reynolds F, Weissleder R. In vivo phage display selection yields atherosclerotic plaque targeted peptides for imaging. Mol Imaging Biol. 2006;8:201-207.

10. Nahrendorf M, Jaffer FA, Kelly KA, et al. Noninvasive vascular cell adhesion molecule-1 imaging identifies inflammatory activation of cells in atherosclerosis. Circulation. 2006;114:1504-1511.

11. Nahrendorf M, Keliher E, Panizzi P, et al. ${ }^{18} \mathrm{~F}-4 \mathrm{~V}$ for PET-CT imaging of VCAM-1 expression in atherosclerosis. JACC Cardiovasc Imaging. 2009;2: 1213-1222.

12. Kelly KA, Allport JR, Tsourkas A, Shinde-Patil VR, Josephson L, Weissleder R. Detection of vascular adhesion molecule-1 expression using a novel multimodal nanoparticle. Circ Res. 2005;96:327-336.

13. Kelly KA, Waterman P, Weissleder R. In vivo imaging of molecularly targeted phage. Neoplasia. 2006;8:1011-1018.

14. Cannistra SA, Ottensmeier C, Tidy J, DeFranzo B. Vascular cell adhesion molecule-1 expressed by peritoneal mesothelium partly mediates the binding of activated human T lymphocytes. Exp Hematol. 1994;22:996-1002.

15. van der Vijgh WJ. Clinical pharmacokinetics of carboplatin. Clin Pharmacokinet. 1991;21:242-261.

16. Markman M, Rothman R, Hakes $\mathrm{T}$, et al. Second-line platinum therapy in patients with ovarian cancer previously treated with cisplatin. J Clin Oncol. 1991;9:389-393. 